\title{
Evaluation of a novel tool for bone graft delivery in minimally invasive transforaminal lumbar interbody fusion
}

This article was published in the following Dove Press journal:

Medical Devices: Evidence and Research

13 May 2016

Number of times this article has been viewed

\section{Jeffrey B Kleiner Hannah M Kleiner \\ E John Grimberg Jr Stefanie J Throlson}

The Spine Center of Innovation, The Medical Center of Aurora, Aurora, CO, USA
Correspondence: Jeffrey B Kleiner The Spine Center of Innovation, The Medical Center of Aurora, PO Box 6287, Denver, CO 80206 Email jeffreybkleiner@gmail.com
Study design: Disk material removed (DMR) during L4-5 and L5-S1 transforaminal lumbar interbody fusion (T-LIF) surgery was compared to the corresponding bone graft (BG) volumes inserted at the time of fusion. A novel BG delivery tool (BGDT) was used to apply the BG. In order to establish the percentage of DMR during T-LIF, it was compared to DMR during anterior diskectomy (AD). This study was performed prospectively.

Summary of background data: Minimal information is available as to the volume of DMR during a T-LIF procedure, and the relationship between DMR and BG delivered is unknown. BG insertion has been empiric and technically challenging. Since the volume of BG applied to the prepared disk space likely impacts the probability of arthrodesis, an investigation is justified.

Methods: A total of 65 patients with pathology at L4-5 and/or L5-S1 necessitating fusion were treated with a minimally invasive T-LIF procedure. DMR was volumetrically measured during disk space preparation. BG material consisting of local autograft, BG extender, and bone marrow aspirate were mixed to form a slurry. BG slurry was injected into the disk space using a novel BGDT and measured volumetrically. An additional 29 patients who were treated with L5-S1 AD were compared to L5-S1 T-LIF DMR to determine the percent of T-LIF DMR relative to AD.

Results: DMR volumes averaged 3.6 $\pm 2.2 \mathrm{~mL}$. This represented $34 \%$ of the disk space relative to AD. The amount of BG delivered to the disk spaces was $9.3 \pm 3.2 \mathrm{~mL}$, which is $2.6 \pm 2.2$ times the amount of DMR. The BGDT allowed uncomplicated filling of the disk space in $<1$ minute.

Conclusion: The volume of DMR during T-LIF allows for a predictable volume of BG delivery. The BGDT allowed complete filling of the entire prepared disk space. The T-LIF diskectomy debrides $34 \%$ of the disk relative to AD.

Keywords: T-LIF, diskectomy, bone graft, lumbar fusion, minimally invasive spinal surgery, pseudoarthrosis

\section{Introduction}

A variety of instrumentation techniques are available to assist with lumbar interbody stabilization. These include different approaches for placing fusion cages (oblique, lateral, anterior, or posterior), using stackable cages, expandable cages, and the application of cage coatings. Although this is a limited inventory, these available inventive strategies do not assist the surgeon with the most vexing problem of interbody fusion: delivery of bone graft (BG) or BG extenders (collectively, BG) to the interbody space.

The chief issue related to the intradiskal transport of most BGs is a mechanical problem. This is due to the consistency of BG material - it is a "compressible fluid" and the pressure applied to it by the plunger of a conventional, end-dispensing bone graft delivery tool (BGDT) (Figure 1) preferentially drives out the liquid part of the mixture, 


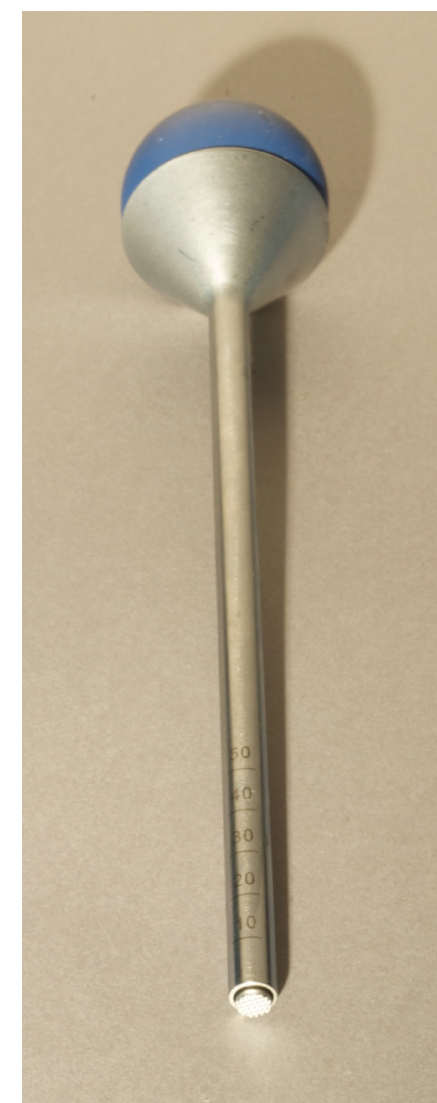

Figure I Standard bone graft delivery funnel.

leaving a condensed plug of the graft material trapped within the cylindrical tool. Removing, clearing, and reinserting the cannula can traumatize the neighboring nerve tissue.

Three other mechanical issues, related to BG delivery with conventional, extended-funnel, round-chambered delivery tools, were identified. The fixed funnel atop the conventional BGDT prevents the surgeon from visualizing the tip of the cannula as it is placed in the disk space annulotomy. This puts the contents of the spinal canal at risk during BGDT insertion.

Next, the tip of the cannula is round and end-dispensing and cannot enter a collapsed disk space without damaging the endplates or skating off to an undesired location. Finally, the conventional, end-dispensing delivery device deposits BG directly in the path of the fusion cage to be applied and does not disperse the graft material into the surrounding, prepared disk space (Figure 2).

Based upon these considerations, a novel BGDT was prototyped with a detachable funnel (Figure 3) and an increased internal cross-sectional area to improve the flow of BG material (Figure 4A and B). The cannula tip was modified to a wedge to allow entrance into a collapsed disk space (Figure 5A-C), and large portals were placed on the sides

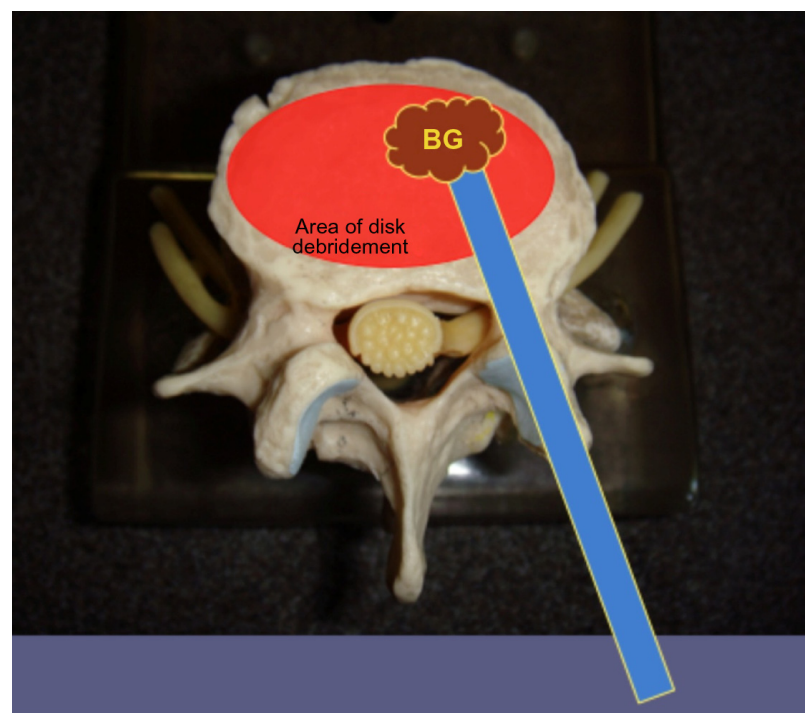

Figure 2 Conventional end-dispensing cannula ejects BG directly in the path of a fusion cage and does not distribute BG into the periphery of the prepared disk space.

Abbreviation: BG, bone graft.

of the cannula to allow the $\mathrm{BG}$ to exit into the prepared disk space out of the way of the fusion cage (Figure 6).

Because it was a modification of an existing surgical tool, not produced for resale or implantation, it was designated an exempt status by the hospital institutional review board to be used by a single surgeon (JBK) and exclusively on transforaminal lumbar interbody fusions (T-LIF) and lateral lumbar interbody fusions.

Initial use of the device revealed that it allowed application of BG and complete filling of the prepared disk space in less than a minute. An interesting trend became apparent after several uses: The amount of BG volume delivered (BGD) to the prepared disk space was more than the amount

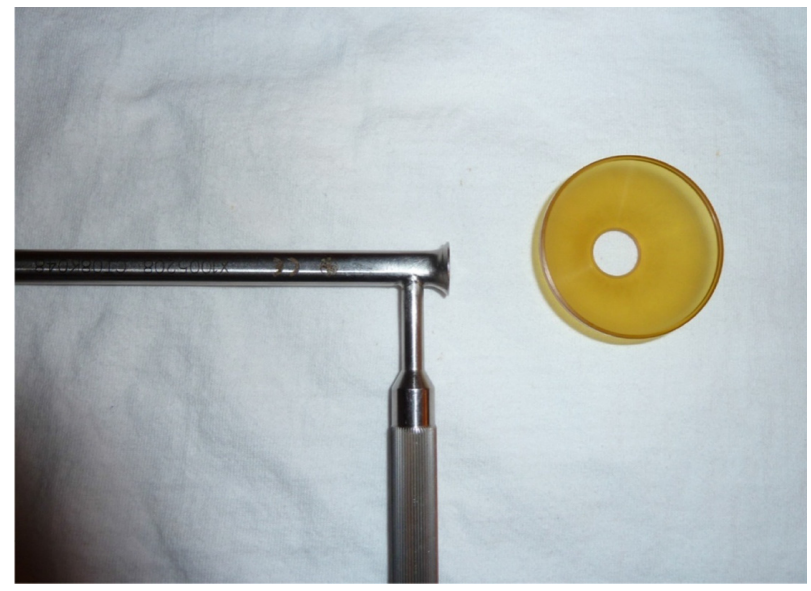

Figure $3 \mathrm{~A}$ removable funnel prevents obscuring the view of the tip of the cannula. 


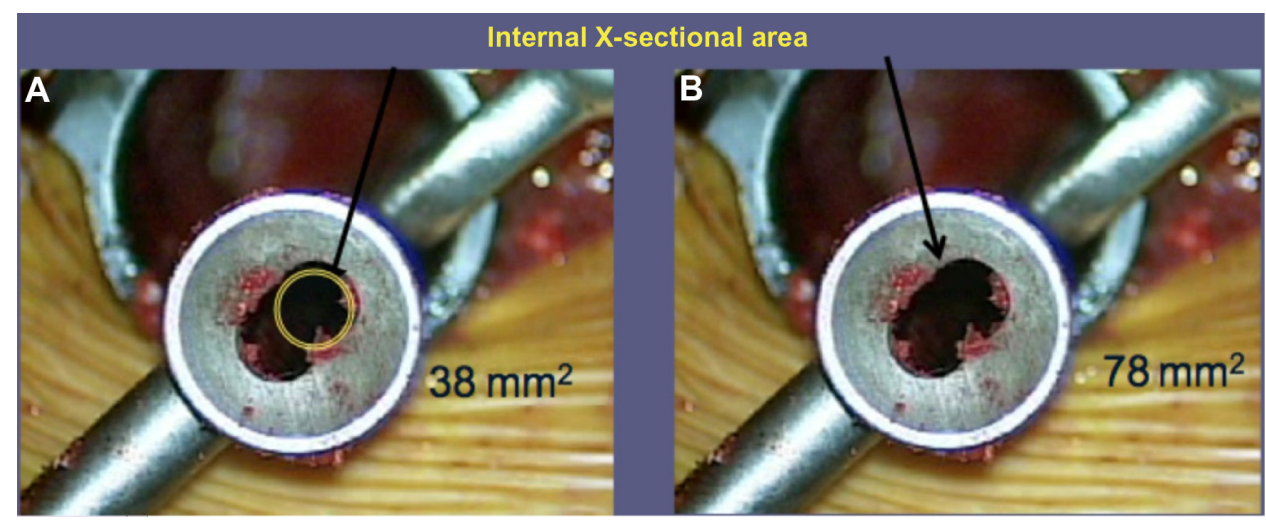

Figure 4 View of the cross section of the bone graft delivery tool relative to the conventional cylindrical tool.

Notes: (A and B) The internal cross-sectional area of the round cannula is only $38 \mathrm{~mm}^{2}$ and is responsible for substantial wall friction relative to the larger, ovoid crosssectional area of the prototype.

of disk material removed (DMR). Unlike the conventional end-dispensing tool, the BGDT did not jam.

The objectives of this study were as follows: 1) to evaluate the amount of DMR during Minimally Invasive Spinal T-LIF surgery, 2) to determine the percentage of DMR removed with this technique relative to a thorough anterior diskectomy, 3) to establish the relationship between DMR and BG delivery, and 4) to assess the safety and utility of a novel BGDT.

Review of the medical literature revealed that no studies have quantified the volume of DMR during MIS T-LIF disk space preparation. Likewise, there are no comparisons of
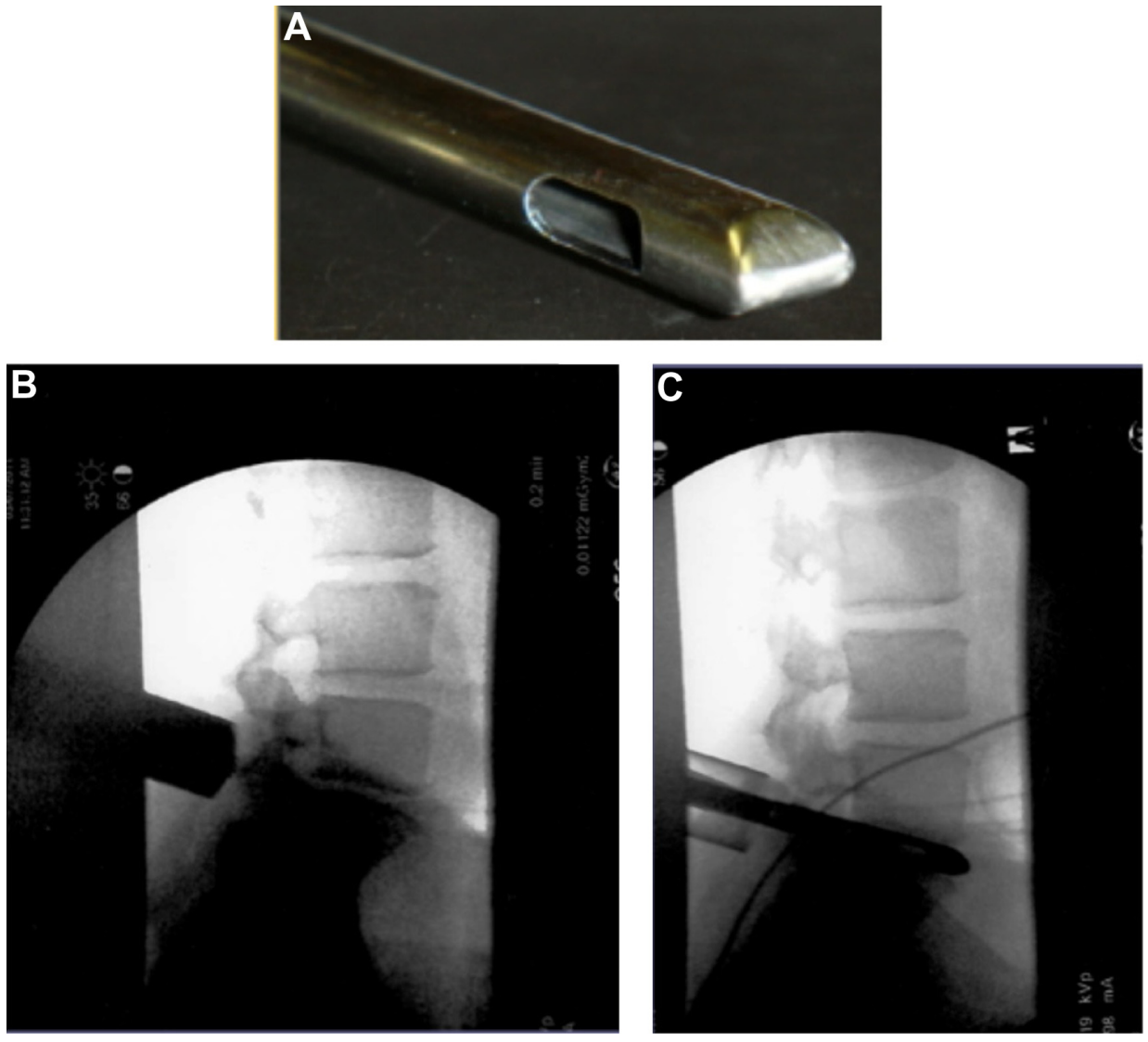

Figure $\mathbf{5}$ Close up of the bone graft delivery tool tip and its radiographic appearance.

Notes: (A) A wedged cannula tip allows entrance into the most collapsed disk space. (B and C) The tapered BG delivery tool takes the path of least resistance and is able to enter even the most collapsed, prepared disk space.

Abbreviation: BG, bone graft. 


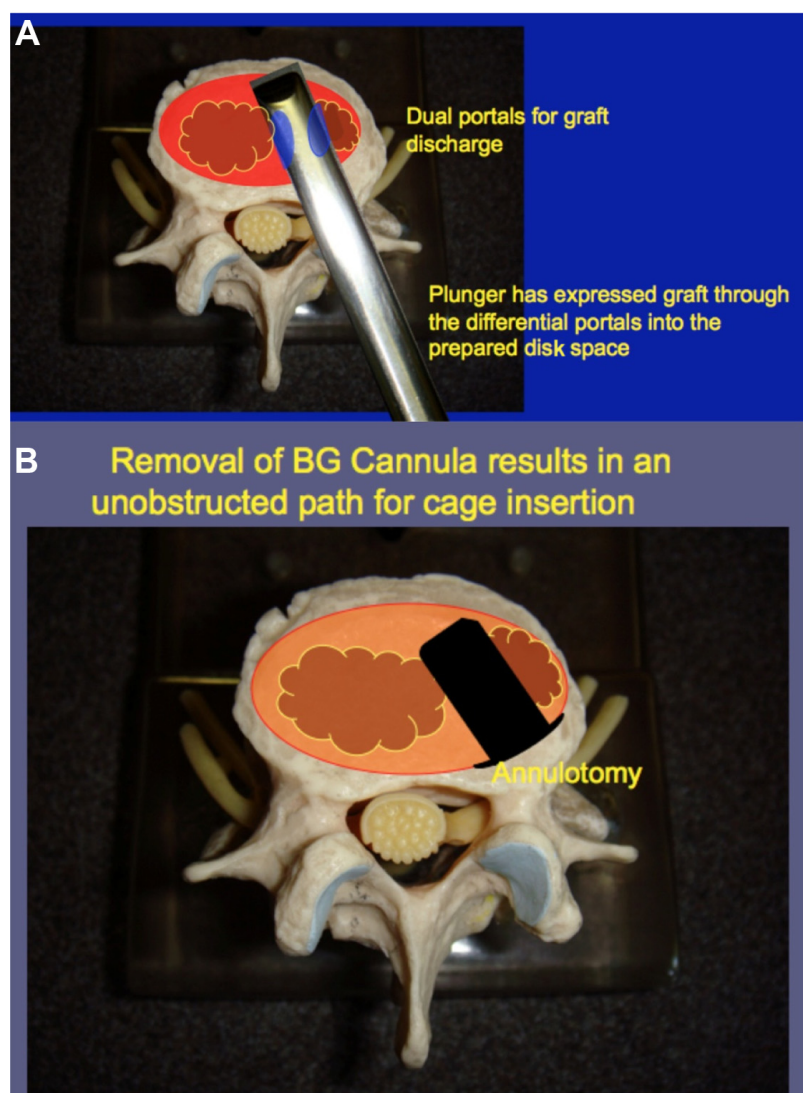

Figure 6 Axial view of the prepared disk space during and after application of the bone graft delivery tool.

Notes: (A) Biportal bone graft delivery tool ejecting bone graft into the prepared area of the disk space. (B) Removal of the BG delivery tool leads to an unobstructed path for a fusion cage.

Abbreviation: BG, bone graft.

the amount of DMR in T-LIF surgery relative to a thorough diskectomy like that performed anteriorly at L5-S1 space. Furthermore, no reports are available as to how much BG or biological material is typically delivered to the T-LIF prepared disk space. Because endplate preparation and BGD volume likely influence successful arthrodesis, an investigation evaluating this relationship is justified.

\section{Materials and methods}

During the time period from July 2010 to December 2012, all patients undergoing MIS T-LIF at the L4-5 and/or L5-S1 levels were studied for DMR and BGD at each disk space during the surgical procedure. The Western Institutional Review Board (WIRB) approved the study. Patient confidentiality and human subjects protocol were scrupulously followed and patients provided written informed consent to be included in the study. The diagnosis was spondylosis or spondylolisthesis in all patients. A total of 65 patients with an average age of 56 years were studied (Tables 1 and 2). There were 31 male and 34 female patients.
Table I Details of the study subjects, L4-5 disk space

\begin{tabular}{|c|c|c|c|c|c|}
\hline BG delivered & Disk removed & Cage volume & Ratio & Age & Sex \\
\hline 10.0 & 2.5 & 0.76 & 4.0 & 46.0 & $M$ \\
\hline 12.0 & 6.0 & 0.91 & 2.0 & 50.0 & $\mathrm{~F}$ \\
\hline 13.0 & 4.5 & $\mathrm{I} .47$ & 2.9 & 71.0 & $M$ \\
\hline 7.8 & 1.8 & 0.56 & 4.3 & 64.0 & $\mathrm{~F}$ \\
\hline 12.5 & 3.0 & 0.91 & 4.2 & 59.0 & $F$ \\
\hline 16.5 & 6.5 & $\mathrm{I} .47$ & 2.5 & 52.0 & $M$ \\
\hline 11.0 & 7.0 & 1.05 & 1.6 & 47.0 & $\mathrm{~F}$ \\
\hline 10.0 & 3.0 & 0.90 & 3.3 & 42.0 & $\mathrm{~F}$ \\
\hline 11.0 & 8.0 & 1.26 & 1.4 & 59.0 & $\mathrm{~F}$ \\
\hline 10.5 & 2.0 & 0.91 & 5.3 & 65.0 & $\mathrm{~F}$ \\
\hline 8.0 & 4.0 & 1.47 & 2.0 & 52.0 & M \\
\hline 13.5 & 3.0 & 0.91 & 4.5 & 50.0 & $\mathrm{~F}$ \\
\hline 14.5 & 1.0 & 0.69 & 14.5 & 43.0 & $M$ \\
\hline 15.5 & 4.0 & 0.91 & 3.9 & 42.0 & $M$ \\
\hline 6.0 & 1.5 & 1.26 & 4.0 & 68.0 & $M$ \\
\hline 13.0 & 4.5 & 1.47 & 2.9 & 54.0 & $\mathrm{~F}$ \\
\hline 14.5 & 5.0 & $\mathrm{I} .47$ & 2.9 & 57.0 & M \\
\hline 14.5 & 7.5 & 1.47 & 1.9 & 74.0 & M \\
\hline 10.5 & 7.5 & 1.67 & 1.4 & 59.0 & M \\
\hline 8.5 & 4.0 & 0.91 & 2.1 & 71.0 & $\mathrm{~F}$ \\
\hline 9.0 & 3.0 & 0.56 & 3.0 & 57.0 & $\mathrm{~F}$ \\
\hline 8.5 & 3.0 & 0.91 & 2.8 & 86.0 & $\mathrm{~F}$ \\
\hline 7.3 & 1.3 & 0.62 & 5.6 & 64.00 & $\mathrm{~F}$ \\
\hline 19.0 & 10.0 & 1.26 & 1.9 & 59.00 & $\mathrm{~F}$ \\
\hline 12.0 & 2.0 & 0.76 & 6.3 & 65.00 & $\mathrm{~F}$ \\
\hline 12.5 & 2.0 & 1.26 & 6.3 & 63.00 & $M$ \\
\hline 7.0 & 3.0 & 1.06 & 2.3 & 70.00 & $M$ \\
\hline 10.5 & 7.0 & I.47 & 1.5 & 79.00 & $M$ \\
\hline 8.0 & 4.0 & 0.91 & 2.0 & 47.00 & $\mathrm{~F}$ \\
\hline 9.0 & 8.0 & 0.91 & I.I & 42.00 & $\mathrm{~F}$ \\
\hline 8.5 & 4.5 & I.47 & 1.9 & 29.00 & $M$ \\
\hline 7.5 & 3.0 & 1.06 & 2.5 & 72.00 & $M$ \\
\hline 14.5 & 7.0 & 1.98 & 2.1 & 69.00 & $M$ \\
\hline 7.5 & 2.5 & 1.06 & 2.5 & 53.00 & $M$ \\
\hline 9.0 & 4.0 & 1.26 & 2.1 & 58.00 & $M$ \\
\hline 6.0 & 5.0 & 0.91 & 1.2 & 35.00 & $\mathrm{~F}$ \\
\hline 7.0 & 5.0 & 0.91 & 1.4 & 58.00 & $\mathrm{~F}$ \\
\hline 9.0 & 5.0 & 1.26 & 1.8 & 40.00 & $\mathrm{~F}$ \\
\hline 7.0 & I.5 & 0.91 & 4.7 & 83.00 & $\mathrm{~F}$ \\
\hline 9.0 & 4.0 & 1.26 & 2.3 & 58.00 & $M$ \\
\hline 8.0 & 3.5 & 0.91 & 2.3 & 41.00 & $\mathrm{~F}$ \\
\hline 9.5 & 3.2 & 0.76 & 3.0 & 60.00 & $\mathrm{~F}$ \\
\hline 10.0 & 4.0 & 0.91 & 2.5 & 40.00 & $\mathrm{~F}$ \\
\hline 13.5 & 9.0 & 1.67 & 1.5 & 64.00 & \\
\hline 8.5 & 5.0 & 1.26 & 1.6 & 61.00 & $\mathrm{~F}$ \\
\hline 7.5 & 3.5 & 0.91 & 2.1 & 80.00 & $\mathrm{~F}$ \\
\hline 6.5 & 1.0 & 0.56 & 6.5 & 85.00 & $\mathrm{~F}$ \\
\hline 6.5 & 1.5 & 1.26 & 4.3 & 54.00 & $\mathrm{~F}$ \\
\hline 5.5 & 3.0 & 0.76 & 1.8 & 36.00 & $\mathrm{~F}$ \\
\hline 9.5 & 6.0 & 1.74 & 1.6 & 54.00 & $M$ \\
\hline 7.0 & 5.0 & 1.26 & I.4 & 50.00 & $\mathrm{~F}$ \\
\hline 11.5 & 3.5 & 1.26 & 3.3 & 57.00 & $M$ \\
\hline 10.5 & 7.2 & 1.67 & 1.5 & 58.00 & $M$ \\
\hline 6.0 & 2.0 & 0.91 & 3.0 & 57.00 & $\mathrm{~F}$ \\
\hline 4.5 & 1.0 & 1.26 & 4.5 & 51.00 & $\mathrm{~F}$ \\
\hline 5.5 & 1.0 & 0.96 & 5.5 & 64.00 & $M$ \\
\hline 7.0 & 4.0 & 1.26 & 1.8 & 59.00 & $\mathrm{~F}$ \\
\hline 10.0 & 3.2 & 1.50 & 3.1 & 66.00 & $M$ \\
\hline
\end{tabular}

Notes: Average BG delivered: $9.8 \pm 3.3 \mathrm{~mL}$. Average disk material removed: $4.1 \pm 2.2 \mathrm{~mL}$. Ratio (BG delivered/disk material removed): $3.1 \pm 2.0$.

Abbreviations: BG, bone graft; $F$, female; $M$, male. 
Table 2 Details of the study subjects, L5-SI disk space

\begin{tabular}{|c|c|c|c|c|c|}
\hline $\begin{array}{l}\text { BG } \\
\text { delivered }\end{array}$ & $\begin{array}{l}\text { Disk } \\
\text { removed }\end{array}$ & $\begin{array}{l}\text { Cage } \\
\text { volume }\end{array}$ & Ratio & Age & Sex \\
\hline 10.0 & 1.0 & 0.45 & 10.45 & 62.00 & $F$ \\
\hline 7.3 & 1.3 & 0.62 & 6.05 & 64.00 & $\mathrm{~F}$ \\
\hline 14.0 & 5.0 & 0.90 & 2.98 & 42.00 & $F$ \\
\hline 19.0 & 10.0 & 1.26 & 2.03 & 47.00 & $F$ \\
\hline 9.5 & 3.0 & 0.76 & 3.75 & 52.00 & $\mathrm{~F}$ \\
\hline 6.0 & 1.0 & 0.76 & 6.76 & 46.00 & $M$ \\
\hline 12.0 & 2.0 & 0.76 & 6.38 & 65.00 & $\mathrm{~F}$ \\
\hline 11.0 & 3.0 & 1.26 & 4.09 & 52.00 & $M$ \\
\hline 7.0 & 1.0 & 0.62 & 7.62 & 50.00 & $\mathrm{~F}$ \\
\hline 5.0 & 1.0 & 0.62 & 5.62 & 43.00 & $M$ \\
\hline 7.5 & 3.5 & 0.91 & 2.42 & 42.00 & $\mathrm{~F}$ \\
\hline 11.0 & 2.0 & 1.06 & 6.03 & 68.00 & $M$ \\
\hline 12.5 & 2.0 & 1.26 & 6.88 & 63.00 & $M$ \\
\hline 8.0 & 4.0 & 0.91 & 2.23 & 71.00 & $\mathrm{~F}$ \\
\hline 7.5 & 1.0 & 0.56 & 8.10 & 59.00 & $M$ \\
\hline 9.5 & 5.0 & 0.91 & 2.10 & 47.00 & $\mathrm{~F}$ \\
\hline 6.5 & 2.0 & 0.91 & 3.71 & 56.00 & $\mathrm{~F}$ \\
\hline 8.0 & 4.5 & 1.26 & 2.06 & 29.00 & $M$ \\
\hline 9.0 & 4.5 & 1.50 & 1.50 & 53.00 & $M$ \\
\hline 7.0 & 3.0 & 0.91 & 2.63 & 35.00 & $F$ \\
\hline 5.5 & 2.0 & 0.76 & 3.13 & 40.00 & $\mathrm{~F}$ \\
\hline 8.0 & 2.0 & 1.06 & 4.53 & 58.00 & $M$ \\
\hline 8.5 & 5.2 & 0.91 & 1.81 & 41.00 & $\mathrm{~F}$ \\
\hline 6.0 & 2.0 & 0.45 & 3.23 & 40.00 & $\mathrm{~F}$ \\
\hline 9.5 & 1.0 & 1.06 & 10.10 & 54.00 & $\mathrm{~F}$ \\
\hline 5.0 & 1.0 & 0.76 & 5.76 & 58.00 & $M$ \\
\hline 6.0 & 3.5 & 1.26 & 2.07 & 57.00 & $M$ \\
\hline 8.5 & 2.5 & 1.06 & 3.82 & 57.00 & $F$ \\
\hline 6.0 & 1.0 & 1.16 & 7.00 & 51.00 & $\mathrm{~F}$ \\
\hline 8.5 & 1.0 & 0.96 & 9.40 & 64.00 & $M$ \\
\hline 10.0 & 4.4 & 1.67 & 2.65 & 55.00 & $M$ \\
\hline 6.0 & 1.0 & 1.06 & 7.01 & 59.00 & $\mathrm{~F}$ \\
\hline 9.0 & 3.0 & 0.76 & 3.25 & 36.00 & $\mathrm{~F}$ \\
\hline 9.0 & 4.5 & 0.97 & 2.22 & 18.00 & $M$ \\
\hline
\end{tabular}

Notes: Average BG delivered: $8.6 \pm 3.1 \mathrm{~mL}$. Average disk material removed: $2.8 \pm 1.9 \mathrm{~mL}$. Ratio (BG delivered/disk material removed): $4.2 \pm 2.4$.

Abbreviations: BG, bone graft; $F$, female; $M$, male.

Ninety-three disk spaces were analyzed. A single surgeon with the same surgical team performed all surgeries. The operations were carried out through a $22 \mathrm{~mm}$ cannula with microscopic control. The midline structures and spinous process attachments were left undisturbed. The disk space was debrided using nonmotorized, hand tools to bleeding subchondral bone. The DMR was measured in a volumetric syringe (Figure 7A). Because the volume of local BG was insufficient to fill the disk space, a preparation of BG material was made. This slurry consisted of silicated tricalcium phosphate granules and hyaluronic acid powder mixed with local $\mathrm{BG}$ and bone marrow aspirate concentrate. The mixture was measured volumetrically (Figure 7B). Disk space mobilization was carried out with serial impaction of distractor tools until appropriate disk height was achieved. Distraction ranged from 8 to $14 \mathrm{~mm}$, with the $12 \mathrm{~mm}$ height being most common.

The BGDT was used to apply the slurry to the disk space. It features a rectangular cross section with the same footprint as a small fusion cage $(8 \mathrm{~mm} \times 12 \mathrm{~mm})$. The tapered tip was placed within the debrided disk space under microscopic control, followed by the application of a snap-on funnel for loading the BG. The BG slurry was then placed in the funnel, and the mixture was injected into the disk space. The
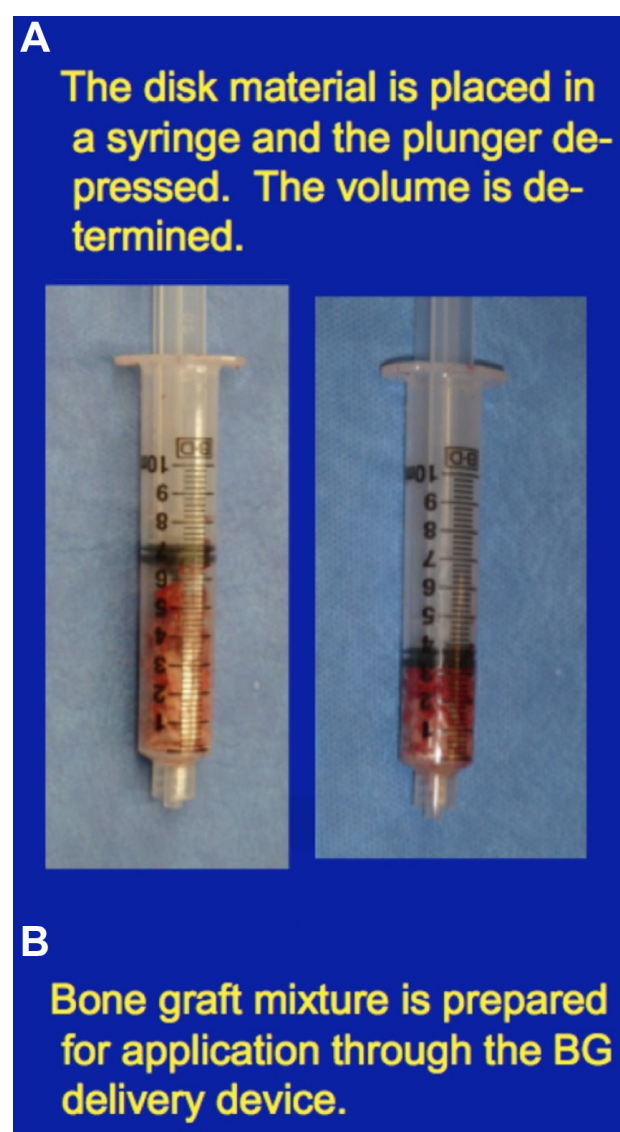

Figure 7 Volumetric measurement of disk material removed and bone graft to be delivered.

Notes: (A) Syringe filled with disk. (B) Bone graft slurry material and compressed for volume measure.

Abbreviation: BG, bone graft. 
biportal design of the delivery tool directed the slurry into the lateral areas of the prepared disk space. Once the disk space was filled entirely, the site of insertion was inspected for any BG material. This material was excluded from the final measurement to ensure an accurate calculation of BGD. Removal of the BGDT provided an unobscured path for fusion cage application (Figure 6A and B).

A hollow polyether ether ketone, interbody fusion cage, of the appropriate size was then placed into the disk space. A minimally invasive, bilateral pedicle screw/rod system was applied prior to wound closure. Average blood loss for the procedures was $127 \pm 75 \mathrm{~mL}$.

The volumes of DMR from L4-5 and L5-S1 were compared to one another, and the volumes of DMR were compared to the volume of BGD from the two disk spaces. A two-tailed Student's $t$-test ( $t$-test) was used to determine whether any significant difference existed between the volumes. The null hypothesis was that no significant difference existed between samples. Significance was set at $P<0.05$.

In order to compare the volume of DMR during a T-LIF procedure with a complete, surgical diskectomy, the volume of DMR during L5-S1 anterior lumbar diskectomy was measured volumetrically in an additional 29 consecutive patients.

The L5-S1 anterior diskectomy is a commonly performed procedure allowing complete diskectomy with visualization and debridement of the entire space and represents an opportunity to calculate the volume of a thorough diskectomy relative to the anatomically and visually constrained T-LIF diskectomy.

The L5-S1 disk was harvested and measured for patients undergoing either anterior fusion or total disk replacement. The DMR consisted of anterior and posterior annulus as well as complete nuclectomy and represented more tissue (in terms of the annuli) than would be typically removed in a T-LIF procedure. There were 29 anterior L5-S1 diskectomy patients. The demographics and diagnosis were similar to the T-LIF patients. There were 16 male and 13 female patients with an average age of 56 years. The diagnosis for this group of patients was spondylolisthesis and or degenerative osteoarthritis with central or lateral recess stenosis.

All study patients were followed with anterior/posterior radiographs and a physical examination at 4 weeks, 12 weeks, 26 weeks, 1 year, and 1.5 years postsurgery. Anterior/ posterior and flexion/extension radiographs were performed on all patients at 1.5 years. All patients who were symptomatic between 1 and 1.5 years postoperatively underwent flexion and extension radiographs and computed tomography (CT) to evaluate for arthrodesis status. A suspicion of pseudoarthrosis was made based upon patient symptoms, radiographs revealing lucency around pedicle screws, lack of graft incorporation, and/or greater than 2.5 degrees of motion on flexion/extension radiographs. A visual analog scale (VAS) for pain was obtained at each visit, and an Oswestry Disability Index (ODI) was completed preoperatively and 1.5 years postoperatively.

\section{Results}

There were 58 L4-5 disk spaces and 35 L5-S1 disk spaces evaluated. The average volumes of DMR from L4-5 and L5-S1 were 4.1 $\pm 2.2 \mathrm{~mL}$ and $2.8 \pm 1.9 \mathrm{~mL}$, respectively (Tables 1 and 2). The $P$-value for the $t$-test was equal to 0.01 , revealing a significant difference in terms of DMR between L4-5 and L5-S1. The comparison between DMR and BGD at L4-5 or at L5-S1 demonstrated a significant difference $(P<0.001)$.

BGD to L4-5 was $9.8 \pm 3.3 \mathrm{~mL}$. At L5-S1, it was $8.5 \pm 3.1 \mathrm{~mL}$. The comparison between DMR and BGD at L4-5 or at L5-S1 demonstrated a significant difference $(P<0.001)$. The $P$-value for the $t$-test was equal to 0.02 , indicating a significant difference in BGD between L4-5 and L5-S1. The combined average was $9.2 \pm 3.0 \mathrm{~mL}$. The amount of DMR compared to the amount of BGD to the disk space was not a 1:1 ratio, as would have been empirically expected. At L4-5, the ratio was 3.1 \pm 2.1 , and at L5-S1, it was 4.2 \pm 2.4 (Figure 8 ). This was statistically significant with a $P$-value of 0.02 . With respect to the entire study, the ratio of BGD

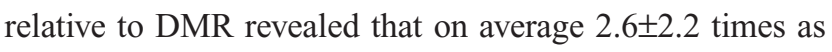
much BG was inserted into the disk space. This finding was even more dramatic with collapsed disk spaces where $1 \mathrm{~mL}$ of DMR led to an average of $6.6 \pm 0.9 \mathrm{~mL}$ of BGD. BGD was asymptotically related to the volume of DMR with $12.3 \mathrm{~mL}$ of $\mathrm{BG}$ being delivered to a disk where $8.0 \mathrm{~mL}$ of disk was removed (Figure 9).

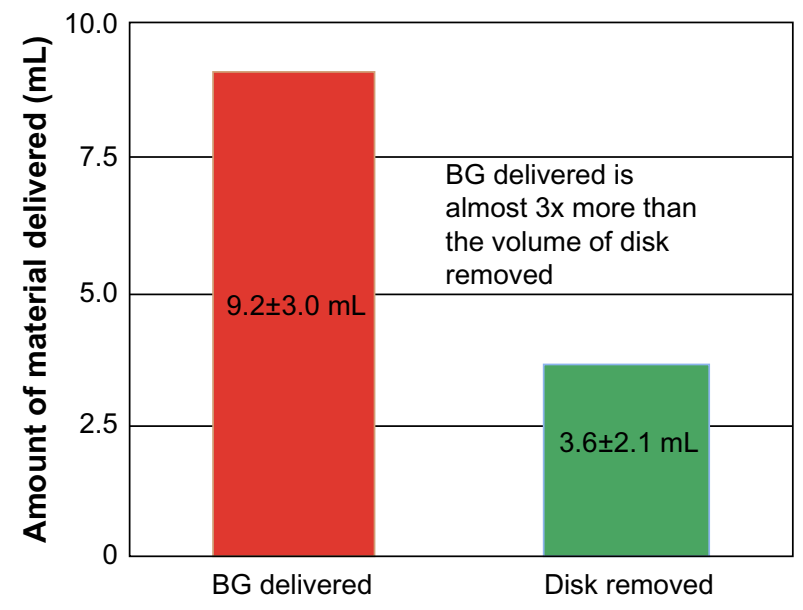

Figure 8 BG delivered versus disk material removed from the L4-SI disk spaces. Abbreviations: BG, bone graft; DMR, disk material removed. 


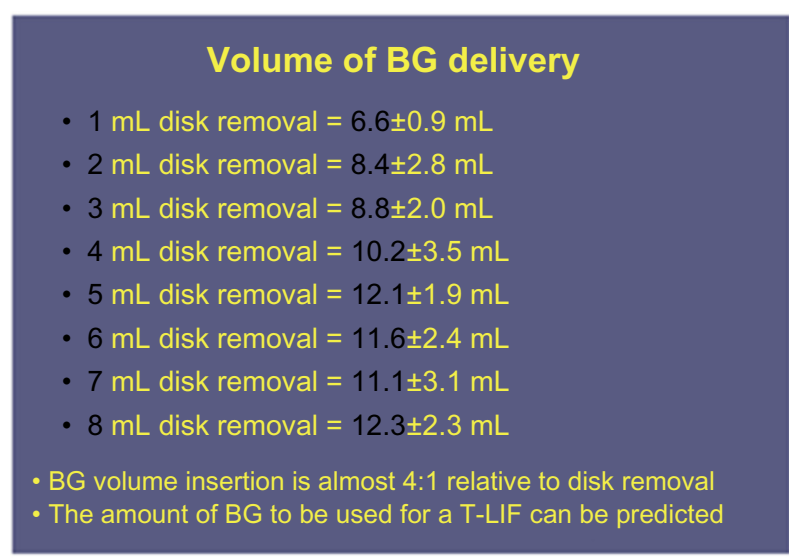

Figure 9 Relationship of BG delivered as a function of DMR

Abbreviations: BG, bone graft; DMR, disk material removed; T-LIF, transforaminal lumbar interbody fusion.

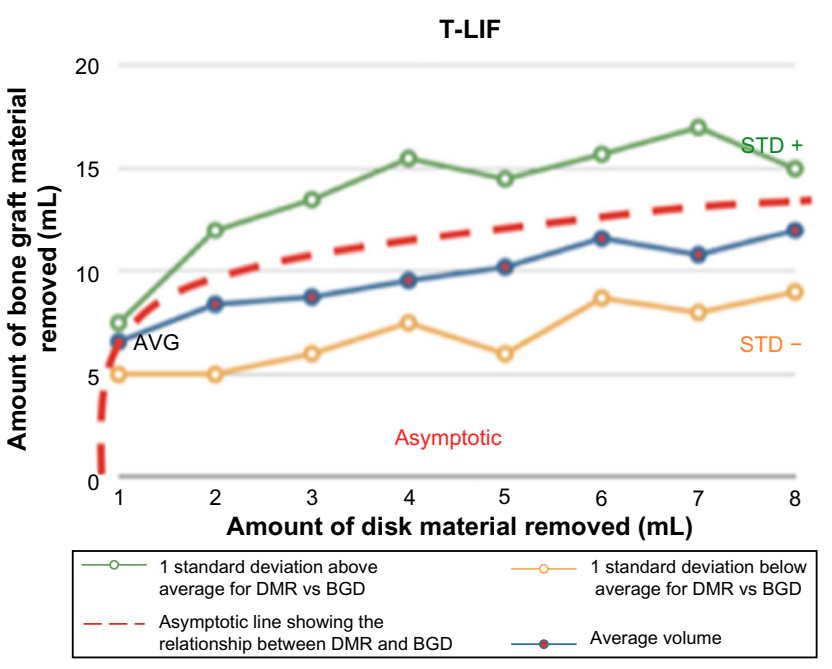

The DMR volume during a T-LIF diskectomy at L5-S1 was $2.8 \pm 1.9 \mathrm{~mL}$, and the average DMR volume from the anterior L5-S1 diskectomy was $8.1 \pm 5.0 \mathrm{~mL}$. Dividing the T-LIF volume by the anterior diskectomy (including annuli) volume revealed that on average DMR via L5-S1 T-LIF was $34 \%$ of the entire disk.

There were no complications associated with the use of the BGDT. Specifically, it did not cause injury to the endplates, penetrate the anterior annulus, jam with BG delivery, or lead to bleeding or infection. It allowed delivery of BG material in $<1$ minute.

The average preoperative ODI measured $29 \pm 9$, and the postoperative value was $21 \pm 8$. A significant difference was not detected with $P=0.06$. The VAS similarly improved with preoperative score measuring $7.8 \pm 1.8$ and postoperative score $4.0 \pm 2.3$ at an average of 18 months. The postoperative VAS was statistically significant relative to the corresponding preoperative value with $P<0.05$.

CT scans were obtained in 26 patients between 1 and 1.5 years postsurgery. Pseudoarthrosis was evident in eight disks and five patients (7.7\%). Two of the patients with a 2-level pseudoarthrosis were hypothyroid. This diagnosis was present in one of the patients with single-level pseudoarthrosis. The other patient with a two-level pseudoarthrosis had a diagnosis of human immunodeficiency virus infection and acquired immune deficiency syndrome (HIV-AIDS). The remaining pseudoarthrosis patient did not have discernible risk factors (diabetes, tobacco consumption, or obesity). A total of eight patients were lost to follow-up, and the average follow-up time was 1.5 years.

\section{Discussion}

There is substantial variation in fusion rates after T-LIF surgery with pseudoarthrosis rates varying from $35 \%$ to $2.9 \% .^{3-7}$ The reasons for the range of successful arthrodesis vary from surgical technique, including $B G$ preparation and application, to the way in which a pseudoarthrosis is diagnosed - direct surgical exploration or by radiographic means.

Reports are available suggesting that bone grafting leading to successful healing is related to dividing cell inoculation. Dallari et $\mathrm{l}^{8}$ showed that rabbit femoral defects inoculated with bone marrow stromal cells yielded a higher percentage of healing than defects treated without bone marrow stromal cells. Hernigou et $\mathrm{al}^{9}$ demonstrated that long bone nonunion in humans could be effectively treated by bone marrow aspiration of autologous iliac crest and that the callus formation was proportional to cell count. While there is no literature to confirm that the volume of BGD to a prepared disk space contributes positively to successful arthrodesis (Figure 10) with inadequate grafting leading to pseudoarthrosis, this is inferred by the long bone studies described above. The importance of sufficient scaffold and viable cellular contribution to a healing bone site are important criteria for healing. ${ }^{8,9}$

Capanna et $a{ }^{10}$ quantized the percentage of disk removal during a diskectomy operation and revealed that an average of $6 \%$ of the disk space was removed. Obviously, the diskectomy technique was not intended to remove the entire disk space or prepare the interspace for fusion. Javernick et a ${ }^{11}$ showed that open, T-LIF diskectomy in a younger, active duty 


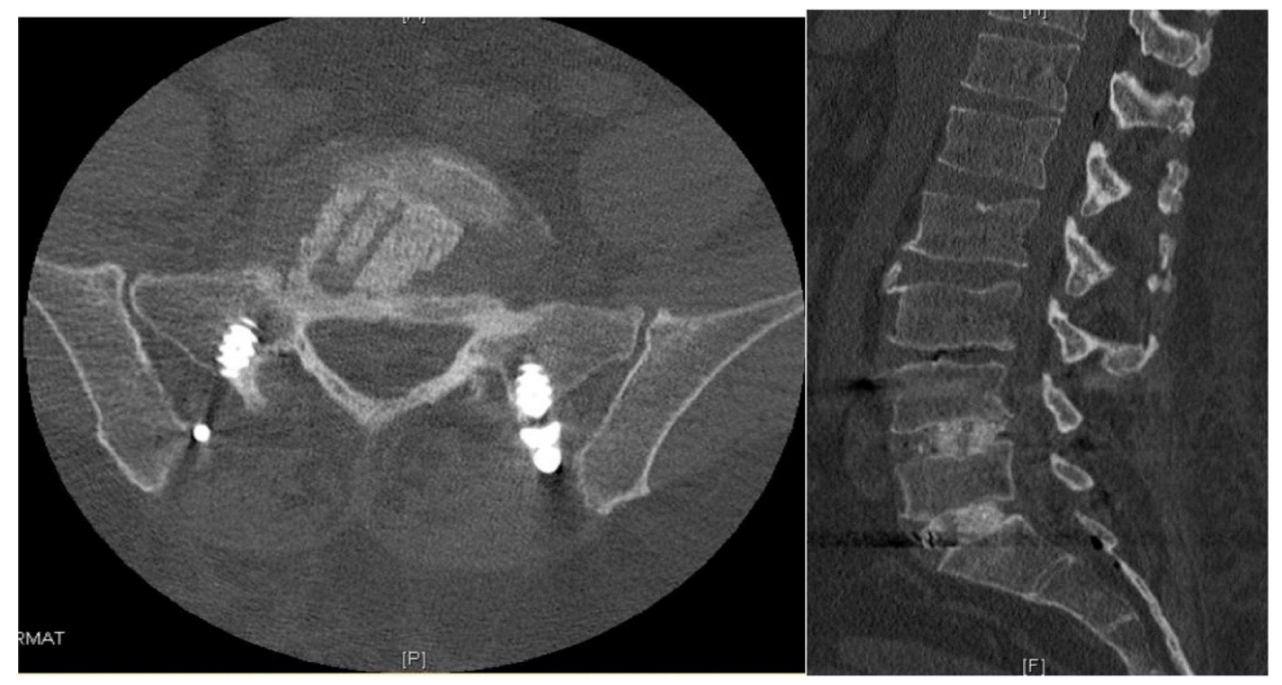

Figure $10 \mathrm{CT}$ scan of the L5-SI disk space showing complete filling of the prepared disk space and successful fusion and incorporation of bone graft I5 months postfusion. Abbreviation: CT, computed tomography.

military population could remove $69 \%$ of the disk. This was an estimate based upon postoperative CT scan. The average age of 36 years, nondegenerative condition of the population, open technique, and a small number of patients (14) likely explain the difference in disk material harvest between their results and those of our report. Using hand tools with minimally invasive T-LIF exposure, a conservative estimate of $34 \%$ of DMR was observed in this study at the L5-S1 level. These differences represent the different goals and/or population of the procedures/patients ${ }^{11}$ and provides a baseline for the expected volume of T-LIF diskectomy during disk space debridement for MIS T-LIF procedures. It also illustrates the critical importance of filling the entire prepared area of the disk space with BG, since just over one-third of the disk space is available for BG inoculation in this particular population of patients.

The statistically significant difference between the amount of DMR from L4-5 versus L5-S1 correlates with the commonly observed radiographic finding of disk height at L4-5 being greater than that of L5-S1. Likewise, BGD to L4-5 was greater relative to the L5-S1 disk space. Although direct volume of BGD was greater at L4-5 relative to L5-S1, the ratio (BG delivered/DMR) was higher at L5-S1 (4.2 \pm 2.4$)$ than at L4-5 (3.1 \pm 2.1$)$. This was a statistically significant difference $(P<0.02)$ and corresponds with the more collapsed disk spaces demonstrating a higher volume of BGD. On average, 2.6 \pm 2.2 times as much BG was applied to the debrided disk space relative to DMR. This is explained by the fact that the disk space was collapsed at the time of diskectomy and then distracted and mobilized during the preparation process to an appropriate height. This is more easily visualized using geometric principles: The disk space can be considered to be a cylinder, the volume of which is $h=\pi r^{2}$. The initial height of the collapsed disk is $h_{1}$ and once distracted is $h_{2}$; this accounts for the increase in disk volume with distraction (Figure 11).

Relying on an empiric 1:1 ratio of DMR to BGD grossly underfills the disk space and is an important contributor to pseudoarthrosis. This is an important consideration in the most collapsed disk spaces since distraction to appropriate height in a noncollapsed disk reduces the ratio to 8:12.3 (Figure 9).

The BG slurry used in this study consisted of a mixture of granular material and liquid. This combination of materials does not behave as a typical, Newtonian (noncompressible) fluid. A non-Newtonian fluid will exude its fluid component as it is compressed, and the residual granular BG material occludes a conventional, cylindrical BG delivery tool.

The BGDT in this study revealed a number of advantages; in that, it allowed for BG application in collapsed disk spaces due to its wedged tip, a process that is not possible with round-ended injection cannulas. The increased

\section{Where does the extra bone graft go?}

The volume of a cylinder $=\mathrm{h} \pi r^{2}$. When the height of the disk is increased by distraction and mobilization, it becomes easy to understand where the additional bone graft goes.
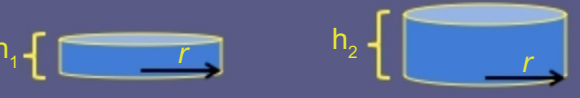

Figure II Geometric explanation for increased volume in a prepared, distracted disk space. 
cross-sectional footprint relative to a round cannula allowed less friction of non-Newtonian fluid material against the walls of the cannula, resulting in an improved BG flow and eliminating jamming due to $\mathrm{BG}$ compression (Figure 4A and B). It is estimated that changing the cross-sectional area from $38 \mathrm{~mm}^{2}$ (internal cross-sectional area of the round cannula) to $78 \mathrm{~mm}^{2}$ (internal cross-sectional area of the BGDT) improves the flow dynamics of a non-Newtonian fluid by $40 \%$. The two sites for BG extrusion at the sides of the cannula tip doubled the exit zone surface area, further decreasing the resistance to flow of the granular mixture. The removable funnel allowed direct visualization of the cannula as it was applied to the disk space without being obscured by the funnel.

The biportal expression of the BG material allowed graft inoculation of all prepared areas of the disk space and left a void for the fusion cage. The applied BG delivery tool allowed refilling of the cannula without having to remove the device, resulting in decreased potential trauma to the adjacent nerve tissue.

The fusion rate in this study was $91.4 \%$ with three of the patients with pseudoarthrosis having a diagnosis of hypothyroidism. This may be related to abnormalities in bone metabolism associated in patients with endocrinopathy. The other two level pseudoarthrosis patients had HIV-AIDS, a known risk factor for pseudoarthrosis. ${ }^{12}$ The other patient did not have apparent risk factors for pseudoarthrosis. According to the criteria used in this paper to assess pain and functional improvement, VAS and ODI, the trend for improvement in these parameters did not reach the standard set $(P<0.05)$ for a statistically significant difference between the preoperative and 1.5 years data point.

A shortfall of this study was that not all patients had a CT scan to confirm arthrodesis status at the end of the study. Only symptomatic patients had this imaging study and had the entire study population been imaged, some patients with asymptomatic pseudoarthrosis could have been detected. An additional concern is that the TCP material has voids between its granules, which can be diminished or collapsed during graft insertion. This could create a discrepancy between the amount of graft measured prior to insertion and the final insertion volume.

In summary, MIS T-LIF preparation of the disk space at L5-S1 can yield $34 \%$ of the disk volume during debridement. BGD was on average 3.5 times the volume of DMR with a relatively higher ratio of $\mathrm{BGD}$ to the more collapsed disk spaces. The described novel BGDT can be used to dispense a volume of BG to the disk space that is capable of filling the entire debrided area in an efficient and safe fashion. This should allow for maximization of arthrodesis potential, increase patient safety, and decrease operative time.

\section{Acknowledgments}

No grant funds were used to support this work. The manuscript submitted does contain information about a noncommercial medical device, which is IRB exempt. This study is approved by the WIRB. JBK and EJG are stake holders in Spinal Surgical Strategies, LLC.

\section{Disclosure}

The authors report no conflicts of interest in this work.

\section{References}

1. Cahill KS, Chi JH, Groff MW, McGuire K, Afendulis CC, Claus EB Outcomes for single-level lumbar fusion: the role of bone morphogenetic protein. Spine. 2011;36(26):2354-2362.

2. Fu R, Selph S, McDonagh, M, et al. Effectiveness and harms of recombinant human bone morphogenic protein-2 in spine fusion. Ann Intern Med. 2013;158(12):890-902.

3. Faundez AA, Schwender JD, Safriel Y, et al. Clinical and radiological outcome of anterior posterior fusion versus transforaminal lumbar interbody fusion for symptomatic disc degeneration: a retrospective comparative study of 133 patients. Eur Spine J. 2009;18(2):203-211.

4. Mehta VA, McGirt MJ, Garcés Ambrossi GL, et al. Trans-foraminal versus posterior lumbar interbody fusion: comparison of surgical morbidity. Neurol Res. 2011;33(1):38-42.

5. Kim JS, Kang BU, Lee SH, et al. Mini-transforaminal lumbar interbody fusion versus anterior lumbar interbody fusion augmented by percutaneous pedicle screw fixation: a comparison of surgical outcomes in adult low-grade isthmic spondylolisthesis. J Spinal Disord Tech. 2009;22(2):114-121.

6. Hee HT, Castro FP Jr, Majd ME, Holt RT, Myers L. Anterior/posterior lumbar fusion versus transforaminal lumbar interbody fusion: analysis of complications and predictive factors. J Spinal Disord. 2001;14(6): $533-540$.

7. Nandyala, SV, Marquez-Lara A, Fineberg, SJ, Singh K. Prospective, randomized, controlled trial of silicate-substituted calcium phosphate versus rhBMP-2 in a minimally invasive transforaminal lumbar interbody fusion. Spine. 2014;39(3):185-191.

8. Dallari D, Fini M, Stagni C, et al. In vivo study on the healing of bone defects treated with stromal cells, platelet-rich plasma, and freeze-dried bone allografts, alone and in combination. J Orthop Res. 2006;24(5):877-888.

9. Hernigou P, Poignard A, Beaujean F, Rouard H. Percutaneous autologous bone-marrow grafting for non-unions. Influence of the number and concentration of progenitor cells. J Bone Joint Surg. 2006;87(7):1430-1437.

10. Capanna AH, Williams RW, Austin DC, Darmody WR, Thomas LM Lumbar discectomy - percentage of disc removal and detection of anterior annulus perforation. Spine. 1981;6(6):610-614.

11. Javernick MA, Kuklo TR, Polly DW Jr. Transforaminal lumbar interbody fusion: unilateral versus bilateral disk removal - an in vivo study. Am J Orthop. 2003;32(7):344-348; discussion 348.

12. Richardson J, Hill AM, Johnston CJ, Norrih AR, Eastwood D and Lavy CB. Fracture healing in HIV-positive populations. $J$ Bone Joint Surg (Br) 2008;90-B:988-994. 


\section{Publish your work in this journal}

Medical Devices: Evidence and Research is an international, peerreviewed, open access journal that focuses on the evidence, technology, research, and expert opinion supporting the use and application of medical devices in the diagnosis, treatment and management of clinical conditions and physiological processes. The identification of novel

devices and optimal use of existing devices which will lead to improved clinical outcomes and more effective patient management and safety is a key feature. The manuscript management system is completely online and includes a quick and fair peer-review system. Visit http://www. dovepress.com/testimonials.php to read real quotes from authors.

Submit your manuscript here: http://www.dovepress.com/medical-devices-evidence-and-research-journal 